\title{
Electrochemical Characterization of the Influence of Scanning Number on the Self-assembled Monolayer Formed by Schiff Base
}

\author{
K. Ding ${ }^{\text {a,b)* }}$, Q. Wang, ${ }^{\text {a) }}$ Z. Jia, ${ }^{\text {a) }}$ R. Tong, ${ }^{\text {a) }}$ X. Wang ${ }^{\text {b) }}$ \\ ${ }^{a)}$ Department of Chemistry, Hebei Teacher's University, Shijiazhuang 050016, China. \\ ${ }^{b)}$ Institute of Coal Chemistry, Chinese Academy of Science, Taiyuan 030001, China
}

Received 27 September 2002; accepted in revised form 18 August 2003

\begin{abstract}
The influence of potential-scan number on the Schiff base self-assembled monolayer was probed by the Cyclic Voltammetry (CV) and Electrochemical Impedance Spectroscopy (EIS) techniques for the first time. The results showed that the charge transferring resistance $\left(R_{c t}\right)$ could be reduced with the potential-scan, suggesting that the structure of the self-assembled monolayer was altered. Also, the relationship between the content of $\mathrm{C}=\mathrm{N}$ group, represented approximately by the reduction electric charge (Qr), and the monolayer's packing degree which was represented by the value of $\mathrm{R}_{\mathrm{ct}}$, was described. Providing a new way to change the interface characterization is the main contribution of this paper.
\end{abstract}

Keywords: number, influence, self-assembled monolayers, Schiff.

\section{Introduction}

As we know, characterizing the self-assembled monolayer has become a key research field, not only due to its potential application in the biochemistry, but also owing to its present application in studying the interfacial problems [1]. In other words, the self-assembly technique has offered a unique opportunity to build a well-ordered dielectric layer between a metal electrode and an

\footnotetext{
* Corresponding author. E-mail address: dkeqiang@263.net
} 
electrochemically active species [1]. Alkanethiols are the main molecules used to generate self-assembled monolayer because of their ease of preparing [1].

But due to lacking of active group, fewer further researches on alkylthiols monolayer were published although their structure is suitable to establish some ideal models for investigating some fundamental problems. Hence, many organic molecules tailored with different functional groups were introduced to create some specific self-assembled monolayer in order to extend the application of self-assembled monolayer. For example, Flink tailored crown ether to the alkylthiol monolayer to detect metal ions, where some interesting results were obtained [2]. Turyan used the self-assembled monolayer of 4-(mercapto-n-alkyl) pyridinium to determine $\mathrm{Cr}(\mathrm{VI})$ ion [3] rationally. In a word, many different organic groups were used to introduce into the self-assembled monolayers, having in mind to observe some interesting phenomena as well as to interpret the interfacial problem in the molecular level.

Meanwhile, the $\mathrm{CV}$ and EIS methods have been employed as a powerful technique to probe the self-assembled monolayer, where some key parameters could be obtained successfully. Kim compared the monolayers formed by different methods, one by self-assembly and the other by Langmuir-Blodgett technique with cyclic voltammograms [4]. Diao discussed the defects in the octadacanethiol self-assembled monolayer successfully with EIS technique [5]. So it is reasonable to believe that some macro-electrochemical behaviour can be observed with $\mathrm{CV}$ and EIS methods although some miro-changes at molecular level could not be explained exactly.

Although the key utilities of Schiff base containing the $\mathrm{C}=\mathrm{N}$ specific group, were found in many reports $[6,7]$, to the best of our knowledge, few reports about the self-assembled monolayer containing $\mathrm{C}=\mathrm{N}$ group were published [8]. The Schiff base, containing the $\mathrm{C}=\mathrm{N}$ group, was first linked into the self-assembled monolayer in our laboratory [9], and found the $\mathrm{C}=\mathrm{N}$ group is still active in the monolayer, therefore, the structure of Schiff base could be varied by the applied electric potential, which will be reported in this paper. 
It has also been reported that in the case of the alkanethiol self-assembled monolayer, the electron transfer rate relates with the chain distance intimately, generally, the longer the molecular chain is, the slower the electron transfer rate is [11]. Therefore, the alkanethiol we introduced into the Schiff base molecule has only two carbons. The Schiff base molecule we used to form the selfassembled monolayer is as follows:

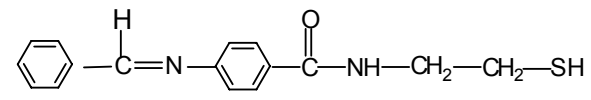

which was synthesized by Peking Capital Normal University and confirmed by NMR and IR spectra.

In this note, we only concentrated on the effect of potential scan number on this novel self-assembled monolayer because of the existence of $\mathrm{C}=\mathrm{N}$ group in this monolayer [10]. The relationship between the charge transfer resistance $\left(\mathrm{R}_{\mathrm{ct}}\right)$, caused by the reduction of $\mathrm{C}=\mathrm{N}$ to $\mathrm{C}-\mathrm{N}$, and the content of $\mathrm{C}=\mathrm{N}$ group represented by the reduction electric charge (Qr), was tried to be established although no linear relationship was found. It has been reported that the charge transfer resistance $\mathrm{R}_{\mathrm{ct}}$ could reflect the packing degree of the self-assembled monolayer [12]; meanwhile the configuration of $\mathrm{C}=\mathrm{N}$ and that of $\mathrm{C}-\mathrm{N}$ is different, hence it is reasonable for us to present the relationship between the content of active groups and the condition of the monolayer, which could be accomplished by studying the influence of potential scan number on the Schiff base self-assembled monolayer.

The meaning of our work is not only to have provided a novel way to study Schiff base, but also a new monolayer where the relationship between the active groups and the monolayer structure can be considered conveniently.

\section{Experimental}

The molecular formula of the Schiff base, which was introduced into the alkylthiol molecule, is $\left(\Phi-\mathrm{CH}=\mathrm{N}-\Phi-\mathrm{CONHCH}_{2} \mathrm{CH}_{2} \mathrm{SH}\right)$, whose structure was 
confirmed by NMR and IR spectra. All other reagents were analytical grade and used as received. All aqueous solutions were prepared with redistilled water.

The cyclic voltammetry (CV) and electrochemical impedance spectroscopy (EIS) measurements were carried out with an ac impedance system (EG\&G, Princeton Applied Research, Model 388) that included a potentiostat/galvanostat (Model 273), a two-phase lock-in analyzer (model 5208), and a person computer. For the EIS measurements, one $5 \mathrm{mV}$ amplitude sine wave was applied to the electrode at open circuit potential. The frequency range adopted was from $0.01 \mathrm{~Hz}$ to 100 $\mathrm{kHz}$, and m388 software was used to carry out the EIS experiment and the results were obtained by Boukamp software [10]. While for the CV experiments, m270 software was used in $\mathrm{CV}$ experiment and the initial potential of the scan was set from the formal potential of the redox couple. All the electrochemical experiments were performed in the traditional three-electrode cell. A large Pt foil and a saturated calomel electrode (SCE) were used as the counter and reference electrode, respectively. The distance between the working electrode and the Luggin-Haber capillary was fixed. All potentials are reported here with respect to SCE. All the experiments were performed at room temperature.

The solution of $1 \mathrm{M} \mathrm{KCl}$ was served as the supporting electrolyte. No other chemicals were introduced into the measuring system.

Self-assembled monolayers of Schiff bases on an Au substrate. Au substrate was prepared on the highly polished side of (111) Si wafers by sputtering with 200 $\mathrm{nm} \mathrm{Au} \mathrm{(99.99 \% ).} \mathrm{To} \mathrm{improve} \mathrm{adhesion} \mathrm{of} \mathrm{Au}$ to the Si wafer, one $10 \mathrm{~nm}$ Ti layer was deposited to the Au substrate. The Au substrates were rinsed with redistilled water and treated with hot and room-temperature Piranha solution $(3: 7,30 \%$ $\mathrm{H}_{2} \mathrm{O}_{2}+$ concentrated $\mathrm{H}_{2} \mathrm{SO}_{4}$ ) for $10 \mathrm{~s}$ and $10 \mathrm{~min}$, respectively. And then the $\mathrm{Au}$ substrate was rinsed with redistilled water and ethanol successively for several times, but the last treatment must be ethanol. And the Au substrate was blown dry with high pure nitrogen, the Au substrate immediately were transferred to $10^{-3} \mathrm{M}$ Schiff base solution of absolute alcohol. The Au electrode was immersed for $24 \mathrm{~h}$ so as to obtain one well-ordered self-assembled monolayer. Before electrochemical measurements, the Schiff base-modified electrodes were washed 
with ethanol and redistilled water successively and then dried with the high pure nitrogen gas successively.

\section{Results and Discussion}

\section{Voltammetry of Schiff base self-assembled monolayer}

The formation of the Schiff base self-assembled monolayer can be testified by the comparison of the cyclic voltammograms between the bare and the monolayer modified electrode as shown in Fig. 1.

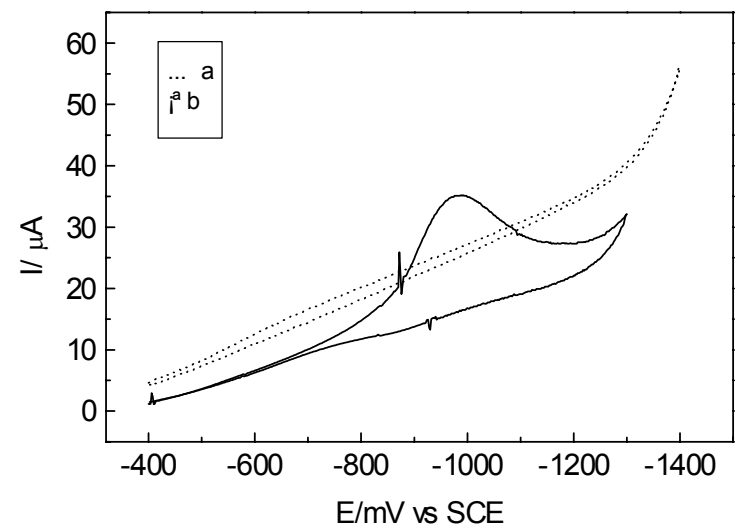

Figure 1. The cyclic voltammograms recorded in $1 \mathrm{M} \mathrm{KCl}$ for different electrodes; a: bare $\mathrm{Au}$ electrode; $\mathrm{b}$ : Schiff bases modified electrode, $\mathrm{V}=100 \mathrm{mV} / \mathrm{s}$.

As can be seen from Fig. 1, for the bare Au electrode represented by line a, no redox peak is observed in the whole potential range from $-0.4 \mathrm{~V}$ to $-1.4 \mathrm{~V}$, except for the reduction peak of $\mathrm{H}^{+}$below $-1.4 \mathrm{~V}$. While for the Schiff base modified electrode, a remarkable reductive peak appears at around $-1 \mathrm{~V}$, accompanied by a small oxidative peak at about $-0.88 \mathrm{~V}$. Evidently, since the $\mathrm{SH}$ group has reacted with the Au substrate to form S-Au bonds as has been confirmed by previous literature [8], there are two active groups $\mathrm{C}=\mathrm{N}$ and $\mathrm{C}=\mathrm{O}$ left in the self-assembled monolayer. It was reported that the bond energy for $\mathrm{C}=\mathrm{N}$ and $\mathrm{C}=\mathrm{O}$ are $173 \mathrm{~kJ} / \mathrm{mol}$ and $532 \mathrm{~kJ} / \mathrm{mol}$, respectively [12]. So it is reasonable to believe that the redox peak for the modified electrode measured in $1 \mathrm{M} \mathrm{KCl}$ solution should be attributed to the $\mathrm{C}=\mathrm{N}$ group in Schiff base selfassembled monolayer. 


\section{The effects of the potential scan number}

Obviously, the reaction of $\mathrm{C}=\mathrm{N}$ group is an irreversible reaction as presented in Fig. 1, so the potential scan number will affect the content of $\mathrm{C}=\mathrm{N}$ group in the Schiff base monolayer directly. The content of $\mathrm{C}=\mathrm{N}$ group will decrease with the potential scan number as seen from Fig. 2, where the reduction peak current dropped dramatically. Interestingly, the attenuation of the reductive current was hardly observed as the scan number exceeds 4 , hinting that the content of $\mathrm{C}=\mathrm{N}$ group in the monolayer was too small to be influenced by the potential scan. What's more, there is no difference observed between the scan numbers 5 and 6 .

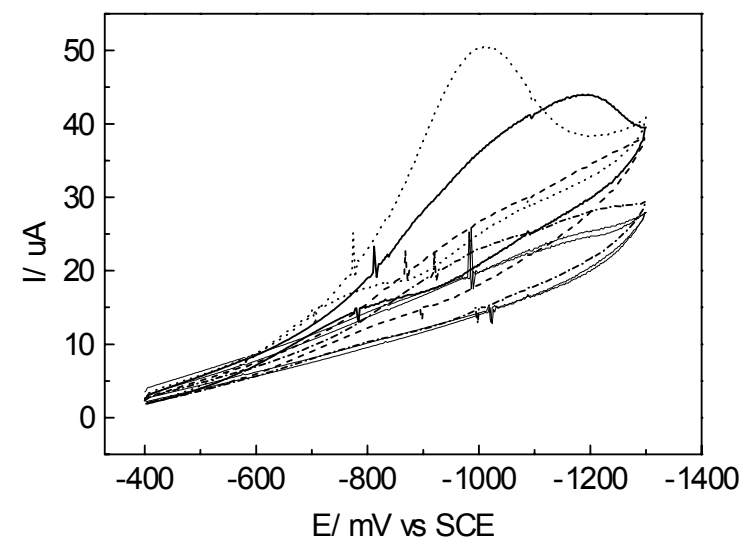

Figure 2. The cyclic voltammograms recorded in $1 \mathrm{M} \mathrm{KCl}$ for the Schiff base selfassembled monolayer modified Au electrode after various scan numbers. From upper to lower is $1,2,3,4,5,6$.

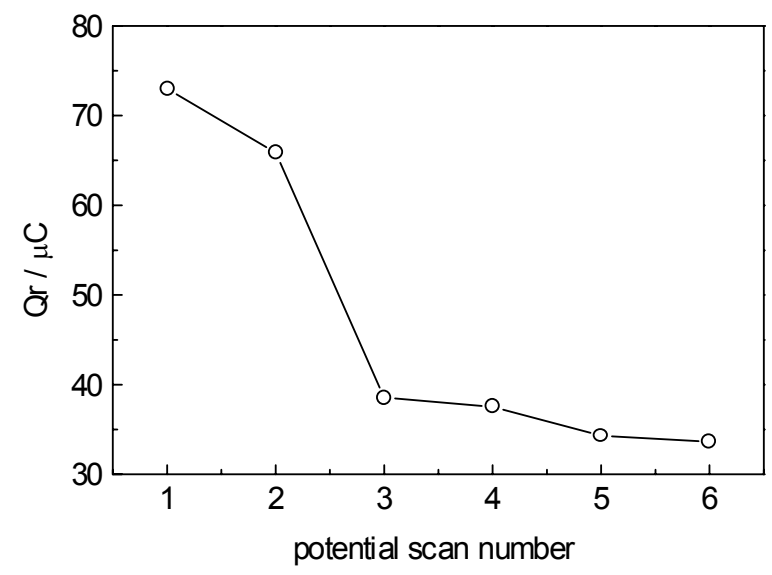

Figure 3. The relationship between reduction electric charge and potential scan number. 
Another interesting thing is that no negative current was exhibited in the whole potential region, though there is a little oxidation peak at about $-0.88 \mathrm{~V}$, implying that the reacting character of $\mathrm{C}=\mathrm{N}$ in this monolayer is rather different from that in the simple Schiff base molecule [13]. From Fig. 3, we know the content of $\mathrm{C}=\mathrm{N}$ group in the Schiff base self-assembled monolayer was reduced with the potential scan number.

It is somehow difficult to measure the content of $\mathrm{C}=\mathrm{N}$ group in the monolayer with our present technique, so we employ the reductive electric charge $Q_{r}$, integrated from the cyclic voltammograms, as a standard to reflect the content of $\mathrm{C}=\mathrm{N}$ group in the Schiff base self-assembled monolayer. Basing on the former report [13], it is very easy to think that the molecular configuration will change as $\mathrm{C}=\mathrm{N}$ was reduced to $\mathrm{C}-\mathrm{N}$ group, hence, the content of $\mathrm{C}=\mathrm{N}$ group will influence the monolayer structure directly. So the novel Schiff base selfassembled monolayer can enable us to investigate the relationship between the content of active group $\mathrm{C}=\mathrm{N}$ and the structure of the monolayer, which is helpful to reconstruct with some specific monolayers. The relationship between the scan number and the reductive electric charge $\mathrm{Q}_{\mathrm{r}}$ is displayed in Fig. 3, from which we know the first potential scan runs, such as from 1 to 3 runs, have a prominent effect on the content of $\mathrm{C}=\mathrm{N}$ group, while for the following runs, from 4 to 6 circles, the influence of potential scan on the content become diminished, telling us the self-assembled monolayer containing redox couple is prone to potential scan.

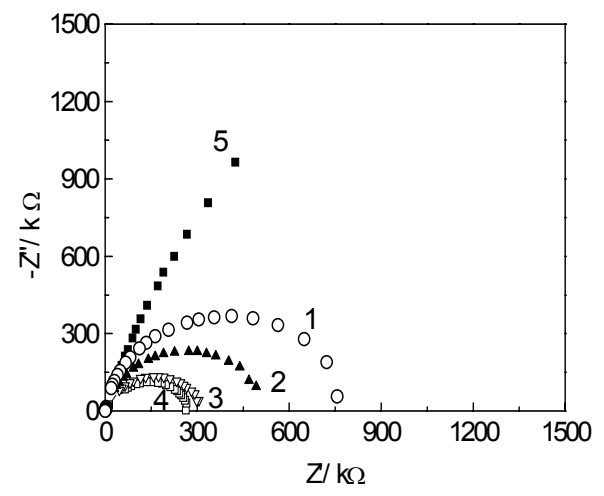

Figure 4. The Nyquist plots for Schiff bases monolayer modified electrode in $1 \mathrm{M} \mathrm{KCl}$ under different scan numbers. The scan numbers are 1, 2, 3, 4, 5 . 


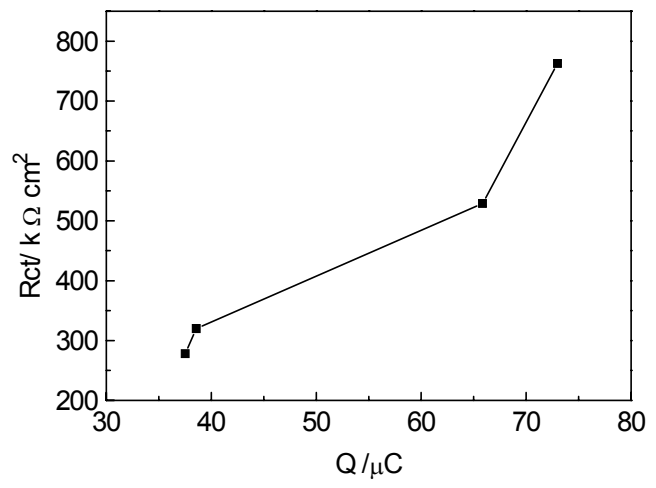

Figure 5. The relationship between the charge transfer resistance Rct and the reductive electric charge Qr when the potential scan number increased.

Fig. 4 presents the Nyquist plots for the Schiff base self-assembled monolayer after different scan number. And the Nyquists were recorded after the cyclic voltammograms. A remarkable semi-circle appears in the whole frequency range as the scan number is 1 . As we know, in the case of the alkanethiol selfassembled monolayer, there is only a line nearly perpendicular to the real resistance axis appearing in the above plot in $\mathrm{KCl}$ solution, where the monolayer could be regarded as a pure capacitance element [11]. Moreover, generally, for the membrane-modified electrode, the semicircle presented in the Nyquist plot corresponds to an equivalent circuit containing a charge transfer resistance parallel to a capacitance element [11], therefore we can only attribute the semicircle to the existence of $\mathrm{C}=\mathrm{N}$ group in this self-assembled monolayer. As seen in Fig. 4, the charge transfer resistance $\left(R_{c t}\right)$, represented by the diameter of the semicircle, becomes smaller as the scan number increases. This phenomenon is opposite to the former formula $\mathrm{R}_{\mathrm{ct}}=\mathrm{RT} / \mathrm{F}^{2} \mathrm{~K}_{\mathrm{app}} \mathrm{C}$, where $\mathrm{C}$ is the redox couple concentration, $\mathrm{K}_{\mathrm{app}}$ the apparent electron transfer rate constant [9]. Theoretically, the concentration of $\mathrm{C}=\mathrm{N}$ group corresponding to $\mathrm{C}$ in the above formula should decrease as the potential scan number increases, the value of $\mathrm{R}_{\mathrm{ct}}$ should become larger. In other words, due to the lacking of active $\mathrm{C}=\mathrm{N}$ group in the monolayer with scan number, the value of $\mathrm{R}_{\mathrm{ct}}$ should get bigger and bigger instead of getting smaller and smaller. But the fact is opposite to our expectation, so the phenomena we observed could not be explained by the former formula simply. 
When the scan number was 5, only a line was left and no semicircle was observed, and the value of $\mathrm{R}_{\mathrm{ct}}$ could not be obtained. As stated usually, the smaller value of $R_{c t}$ corresponds to a looser monolayer structure [11]. As for our studied Schiff base self-assembled monolayer, we have proved that the monolayer gets looser and looser when the scan number increases, which was proved by other cyclic voltammetric experiment where the redox couple of $\mathrm{Fe}(\mathrm{CN})_{6}^{3-/ 4-}$ was used as the probing pin [10].

Fig. 5 is the plot of $R_{c t}$ versus the reductive electric charge, in which $R_{c t}$ becomes smaller and smaller as the content of $\mathrm{C}=\mathrm{N}$ group decreased. Because when the potential scan number is 5 , the value of $R_{c t}$ could not be obtained, there is only 4 points exhibited. Probably the following reasons can explain the above results to some extent. With the potential scan, most molecular configurations were verified due to the reduction of the active group $\mathrm{C}=\mathrm{N}$ to $\mathrm{C}-\mathrm{N}$ group; as a result, the order degree of the monolayer was damaged and the monolayer became looser. It is very interesting that when the scan number was up to 5 , the semicircle in the above plot disappeared, suggesting that the structure of this selfassembled monolayer has been changed and the monolayer could not be regarded as a pure capacitance. The phenomena observed from this novel self-assembled monolayer are rather distinguished from the alkylthiol self-assembled monolayer, which is meaningful to create novel self-assembled monolayer. The detailed experiments will be done in other reports.

\section{Final Comments}

The results in this note mainly demonstrated that the structure of the novel Schiff base self-assembled monolayer was influenced by the potential scan remarkably, which is meaningful to study the relationship between the active groups and the characterization of self-assembled monoalyer. Here we created a self-assembled monolayer where the functional group was introduced in the middle part of the monolayer rather than at the end. Secondly we developed a new way to investigate the relationship between the electric field and the monolayer structure, that is to say, we can change the interfacial features through controlling 
the applied electric potential. Thirdly, the results could not be interpreted by the previous theory simply, hinting the novel system is rather different from the selfassembled reported before. Of course, we must admit that explanations on the above results still remain unclear although the results are very interesting and some more sensitive techniques are really needed to observe this monolayer at molecular level. Further investigations are in progress.

\section{References}

1. A. Ulman, Chem. Rev. 96 (1996)1533.

2. S. Flink, F.C.J.M. Veggel, D.N. Reinhoudt, J. Phys. Chem. B, 103 (1999) 6515.

3. I. Turyan, D. Mandler, Anal. Chem. 69 (1997) 894.

4. Y.H. Kim, Y.T. Kim, Langmuir 15 (1999) 1876.

5. P. Diao, M. Guo, R.T. Tong, J. Electroanal. Chem. 495 (2001) 98.

6. I.M.A. Awad, Rahman, E.A. Bakite, J. Chem. Tech. Biotechnol. 51 (1991) 183.

7. T.X. Wu, Z.J. Li, J.C. Zhao, J. Chem. Chin. Univ. 19 (1998)1617.

8. K.Q. Ding, Z.B. Jia, Q.F. Wang, N. Tian, R.T. Tong, X.K. Wang, H.B. Shao, Chin. Chem. Lett.12 (2001) 1101.

9. E. Sabatani, I. Rubinstein, J. Phys. Chem. B, 91 (1987) 6663.

10. K.Q. Ding, Q.F.Wang, Z.B. Jia, N. Tian, et al., J. Chin. Chem. Soc. 49 (2002)185.

11.P. Diao, D.L. Jiang, X.L. Cui, et al., J. Electroanal. Chem. 464 (1999) 61.

12. Handbook of Chemistry and Physics, CRR Press Inc., F233.

13. C. Gluchowski, L. Cooper, D.E. Bergbreiter, M. Newcomb, J. Org. Chem. 45 (1980) 3413. 